\title{
Taking it out of context: The role of contextual coherence during social event segmentation
}

\author{
Nida Latif ${ }^{1} \cdot$ Francesca Capozzi $^{1}$ - Jelena Ristic ${ }^{1}$
}

Published online: 28 May 2019

(C) The Psychonomic Society, Inc. 2019

\begin{abstract}
Social event segmentation, or parsing of the ongoing dynamic content into discrete social events, is thought to represent a mechanism that supports the expert human ability to navigate complex social environments. Here, we examined whether this ability is influenced by the temporal coherence of the context and by different sources of perceptual information. To do so, we created two video clips, one in which several situations unfolded in a contextually consistent manner, and the other in which the order of these situations was scrambled using a random sequence. Participants viewed each clip and were asked to mark social and nonsocial events in counterbalanced blocks of trials. We analyzed key-press behaviour as well as visual and auditory signals within the clips. Results showed that participants agreed on similar social and nonsocial events regardless of context availability, with greater agreement for social relative to nonsocial events. Context, however, modulated the reliance on sources of perceptual information, such that visual and auditory information was used differently when context was unavailable. Together, these data show that contextual coherence does not determine social event segmentation but serves a modulatory role in perceivers' reliance on perceptual sources of information when identifying events in complex social environments.
\end{abstract}

Keywords Social event segmentation $\cdot$ Event segmentation $\cdot$ Context $\cdot$ Perceptual cues

Humans constantly interpret complex and dynamically changing environments. Studies show that one way in which we manage this large perceptual demand is by grouping information into meaningful events. For example, when observing an individual making a bed, we might identify three distinct events: when the individual (1) takes off the sheet, (2) takes off a pillowcase, and (3) puts on a new sheet. It is well established that observers demonstrate remarkable agreement when they are asked to segment events in this manner (Zacks \& Swallow, 2016). Such segmentation behaviour is thought to be guided by changes in both environmental features, like motion (Zacks, 2004), as well as contextual factors, such as perceived causal relationships between actors and objects (Speer, Reynolds, \& Zacks, 2007) or overall action goals (Magliano, Miller, \& Zwaan, 2001).

Nida Latif

nida.latif@mail.mcgill.ca

1 Department of Psychology, McGill University, 2001 McGill College Ave., Montreal, QC H3A 1G1, Canada
Recent work shows that humans group social information, such as situations involving people, in a different way than nonsocial information, such as situations containing inanimate objects. Boggia and Ristic (2015) were among the first to demonstrate this finding using a segmentation procedure. In their experiment, they presented participants with a video depicting a naturalistic interactive scenario and asked participants to indicate with key presses, in separate blocks, when one social or nonsocial event ended and another one began. Participants showed greater response agreement when marking social relative to nonsocial events while their attentional allocation during the social, but not the nonsocial task, was more focused on social cues. This behaviour during social segmentation was more similar to passive viewing, which was completed at the start of the session, suggesting that segmenting social events likely involved different underlying processes than segmenting nonsocial events. In particular, greater response agreement during social segmentation and attention paid to social cues (i.e., people's eyes and faces during the passive viewing) demonstrated that perceivers distinguish social events in a more naturalistic, automatic, and implicit manner relative to nonsocial events. In the present study, we investigated what role the overall context and perceptual information available within the clip play in this segmentation process. 
Contextual information influences perception and behaviour in many different ways, from basic perception (e.g., the apparent lightness of an object is determined by the context in which it is embedded; Anderson \& Winawer, 2005) to recognizing actions towards particular goals (e.g., preforming an action in a contextually consistent setting such as cracking an egg in a kitchen; Wurm \& Schubotz, 2012). Here, we examined how context, defined as the temporal sequence of situations within the clip, which also provided the clip's narrative coherence, influenced participants' parsing of social and nonsocial events. Studies of nonsocial event segmentation show that perceptual events are often comprehensible even when events are presented in out-of-order temporal sequences. This is taken as a demonstration of the automatic and fundamental nature of these perceptual units. For example, when context is eliminated by presenting events in reverse temporal order, participants are still consistent in identifying event boundaries, showing that overall context contributes little to nonsocial event segmentation (Hard, Tversky, \& Lang, 2008). However, it is at present unknown if the same disruption of temporal context also spares social event segmentation. Unlike nonsocial events, social information changes dynamically and varies over time, with the general context often providing critical information for appropriate interpretation and actions (e.g., Pelphrey, Morris, \& McCarthy, 2004). Thus, one question that we examined here is whether removing the appropriate temporal context affected social and nonsocial segmentation in a similar way.

Furthermore, different perceptual sources make distinct contributions towards contextual information. It is known that in noisy or ambiguous social environments, perceivers may alter their reliance on different sources of perceptual information to make accurate social inferences and decisions. In studies of communication, for example, attending to the head motion of a talker plays a particularly important role in improving perception of speech in contexts with auditory noise (Munhall, Jones, Callan, Kuratate, \& Vatikiotis-Bateson, 2004), whereas listening to speech can improve emotion recognition when the visual facial expressions are ambiguous (de Gelder \& Vroomen, 2000). As such, the second question that we examined here is whether the lack of an appropriate context modulated perceivers' reliance on available perceptual visual and auditory cues. Specifically, we investigated the amount of motion in the visual domain and the intensity of the sound in the auditory domain, as each have been previously shown to play an important role in facilitating perception within complex social settings (de Gelder \& Vroomen, 2000; Munhall et al., 2004).

To address these two questions, we asked participants to view and segment two video clips into social and nonsocial events. One video was the same as that used in the original Boggia and Ristic (2015) study. This video contained the natural broader context with 12 situations presented temporally within a coherent story line (i.e., the contextual version). The second video contained the same situations, but the context was removed by temporally scrambling these situations using a random number sequence (i.e., the noncontextual version).

If context mediated the ability to perform social segmentation, we expected to find lower response agreement for social relative to nonsocial event segmentation in the noncontextual relative to the contextual condition. Further, if in impoverished contexts participants rely on different sources of perceptual information, we expected that the relationship between participants' segmentation responses and available perceptual cues would vary across contextual and noncontextual conditions.

\section{Method}

\section{Participants}

One hundred and ninety-five $(N=195)$ undergraduate students participated in the study (152 females; $M_{\text {age }}=20.54$ years, $S E=.13)$. Mirroring previous studies on event segmentation (e.g., Magliano \& Zacks, 2011; Zacks, 2004), this sample size was determined a priori to ensure about 100 participants per contextual condition presentation order $(N=100$ noncontextual condition first; $N=95$ contextual condition first; see Design and Procedure). Participants were recruited through the psychology volunteer participant pool and received course credits for their participation. The study was completed online and took approximately 60 minutes. This project was approved by the University Research Ethics Board, and all participants provided written informed consent. Additional informed consent was obtained from actors whose identifying information was used in the creation of the stimuli.

\section{Apparatus and stimuli}

The stimulus was an 8-11-minute video that comprised 12 situations. These situations were independently standardized for length (to an average of $45 \mathrm{~s}$; range: $30 \mathrm{~s}-63 \mathrm{~s}$ ) and the number of people present $(0,1$, or 2$)$. Situations comprising the video were selected such that there were two social and two nonsocial situations, each containing zero, one, and two people.

Two versions of the video were created, a contextual and a noncontextual one. Visual depictions of the two conditions are illustrated in Fig. 1a-b. Their time line is shown in Fig. 3. Both videos were filmed in a laboratory, independently, with constant lighting, and without editing cuts. It should be noted that while the situations were standardized to be within the same duration range, there were minor differences in situation duration between the two versions due to independent filming. In the contextual version, the order of situations was follows, with the duration of each situation indicated in brackets: 


\section{a Contextual Condition}

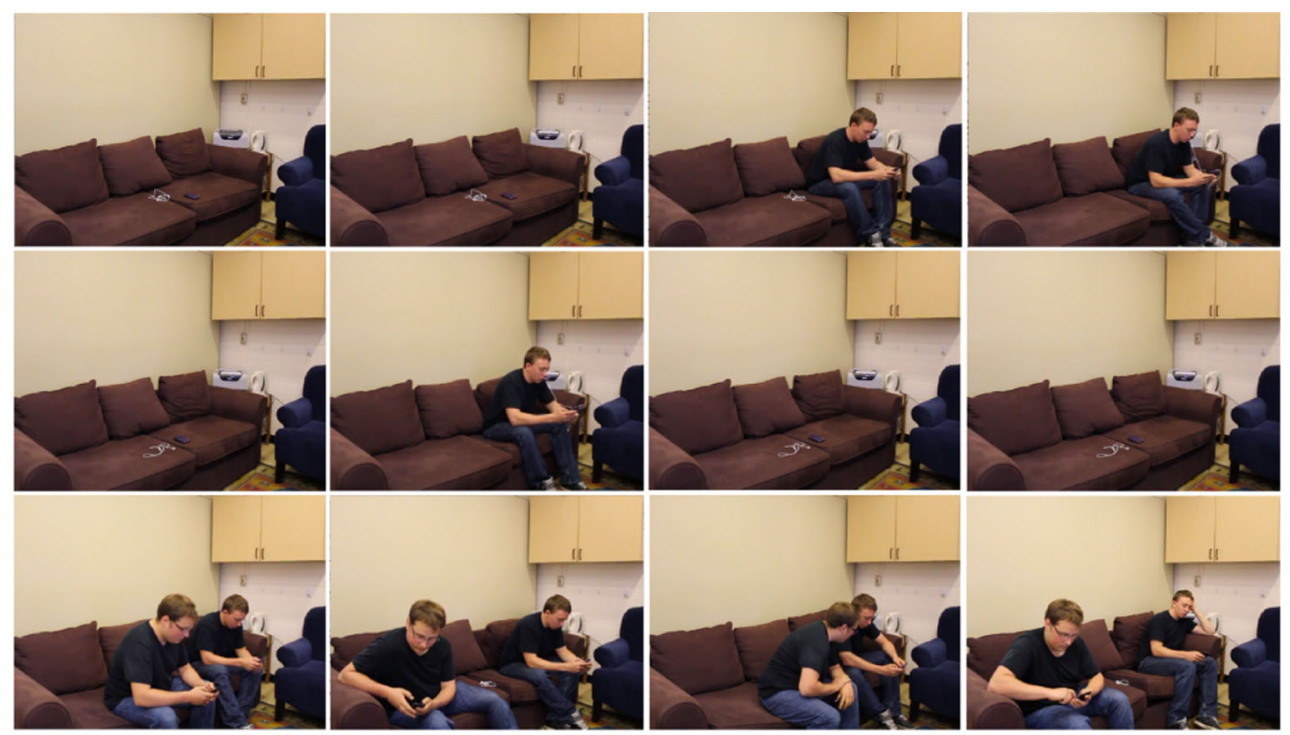

\section{b Non-Contextual Condition}

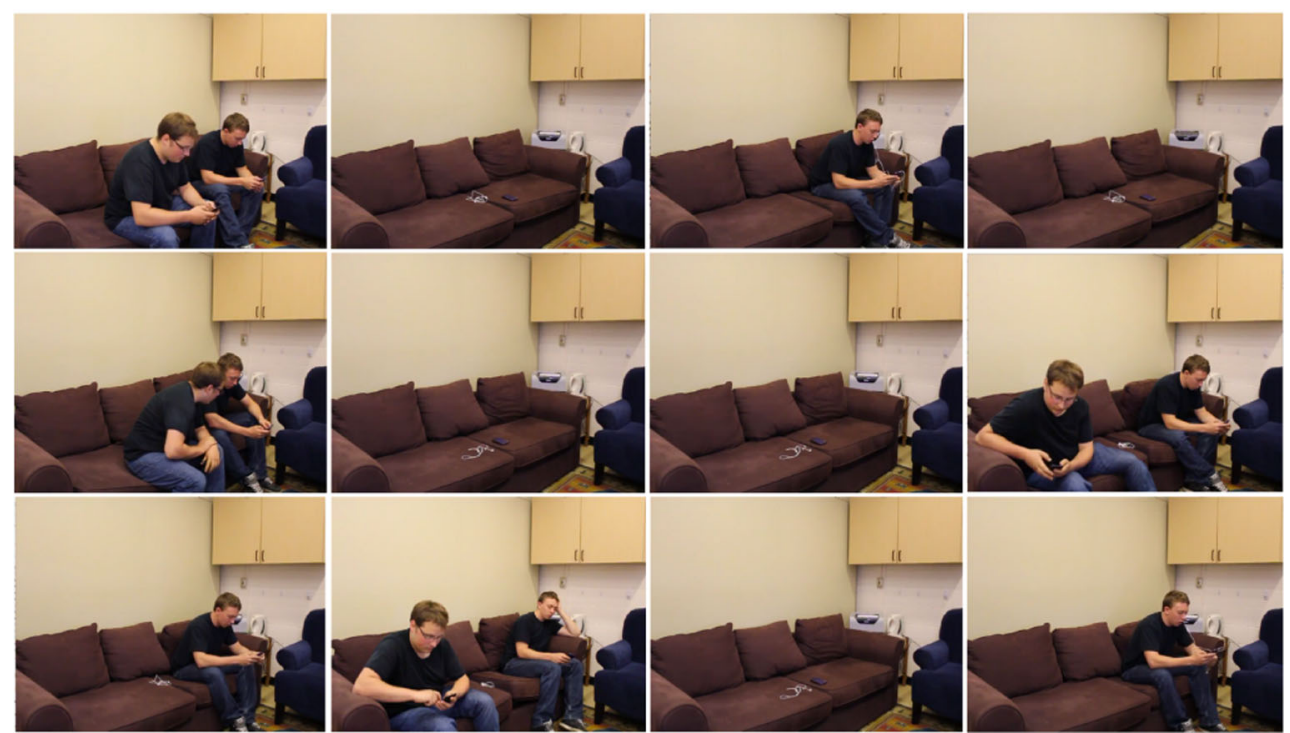

Fig. 1 Video still frames depicting situation order in the contextual (a) and the noncontextual clip (b)

(1) A phone sitting on a couch receives a missed call (56 s). (2) A printer prints a sheet of a paper (40 s). (3) Actor 1 enters the room, sits on a couch, and reviews his weekly class schedule out loud (42 s). (4) Actor 1 then goes over a grocery list out loud ( $37 \mathrm{~s})$. Actor 1 leaves the room. (5) Actor 1 returns a missed call from his mother and leaves a voicemail ( $35 \mathrm{~s})$. (6) Actor 1 leaves the room and receives another missed call (30 s) (7) A fax machine sounds and receives a fax (35s). (8) Actor 1 returns to the room and talks to his mother on the phone about groceries (34 s). (9) Actor 1 begins playing a game on his phone. Actor 2 enters the room and the two actors play online sudoku individually (49 s). (10) Actor 2 begins playing a different game (solitaire) individually (63 s). (11) Actor 2 asks to play sudoku with Actor 1, which the two actors play together on the same phone as they discuss possible moves (37s). (12) When the game is finished, the two actors continue to play different games individually. Actor 1 falls asleep (53 s).

In the noncontextual version, these same situations were reordered using a randomly generated number sequence so that situations no longer depicted a coherent story line. The 
video was then re-recorded in a single take with no editing cuts, following the randomly generated reordered sequence. The order of the situations in the noncontextual condition, illustrated in Fig. 1b, with the duration of each situation indicated in brackets was as follows:

(1) Actor 1 enters, sits on a couch, and begins playing a game on his phone. Actor 2 enters the room, and the two actors play online sudoku individually. The actors leave the room (58 s). (2) A printer prints a sheet of a paper (30 s). (3) Actor 1 returns to the room and talks to his mother on the phone about groceries. Then Actor 1 leaves the room (40 s). (4) A phone sitting on a couch receives a missed call ( $45 \mathrm{~s})$. (5) Actor 1 returns to the couch and plays on his phone. Actor 2 enters and asks to play sudoku with Actor 1, which the two actors play together on the same phone as they discuss possible moves. Actor 2 leaves the room (47 s). (6) Actor 1 then goes over a grocery list out loud. Actor 1 leaves the room (30 s). (7) A fax machine sounds and receives a fax (30 s). (8) Actor 1 returns to the couch and plays on his phone. Actor 2 enters and begins playing a different game (solitaire) individually. Actor 2 leaves the room (35 s). (9) Actor 1 returns a missed call from his mother and leaves a voicemail (58 s). (10) Actor 2 returns to the room and the two actors continue to play different games individually. Actor 1 falls asleep. Actor 2 leaves the room (43 s). (11) Actor 1 wakes up, leaves the room, and receives another missed call (32 s). (12) Actor 1 enters the room, sits on a couch, and reviews his weekly class schedule out loud (42 s).

The experiment was coded in JavaScript using jsPsych, a library developed to run behavioural experiments in a web browser (de Leeuw, 2015; see sample code on Open Science Framework: https://osf.io/js87d). The videos were presented at 60 frames per second at a resolution of $640 \times 480$ pixels.

\section{Design and procedure}

The study was presented such that condition (passive viewing, social segmentation, nonsocial segmentation) and context (contextual, noncontextual) were manipulated within subjects. Participants viewed each clip six times - three times for the contextual and three times for the noncontextual conditions. Order of presentation was counterbalanced such that half the participants $(N=100)$ viewed the noncontextual version first, while the other half $(N=95)$ viewed the contextual version first. Passive viewing, as in Boggia and Ristic (2015), always occurred at the start of the session and was included to familiarize participants with the video. Passive viewing is commonly included to ensure that the data do not reflect differences in video comprehension, but rather the effects of the manipulation itself (e.g., Kurby \& Zacks, 2008). For passive viewing, participants were instructed to "please watch the video carefully and remember as much as you can. There is no need to respond or press any keys." Then, they were presented with the videos again and asked to segment each one into social and nonsocial events, in a counterbalanced order. They were instructed as follows: "The following video contains various events. Please watch the video and press the spacebar when you believe one social/nonsocial (depending on the condition) event ends and a new one begins."

Participants were presented with written instructions prior to each video indicating whether they should watch the following clip passively, mark social events, or mark nonsocial events. Participants controlled the start of each video and were instructed that once the trial began, they would be unable to pause the video.

\section{Results}

\section{Event segmentation}

To be consistent with Boggia and Ristic (2015), key-press responses were binned into 15 -second intervals by summing all responses within each of those time bins for the length of the clip. ${ }^{1}$ The mean and standard deviation of response frequencies for each bin were calculated and standardized to $z$ scores. The between-participant agreement in identifying event boundaries or 'break points' was determined by identifying bins at which the total number of key presses exceeded one standard deviation $(S D)$ of the group mean across all bins. This method of group-based identification of events has previously been shown to provide reliable estimates of overt event segmentation behaviour (Newtson \& Engquist, 1976; Zacks, Swallow, Vettel, \& McAvoy, 2006).

Figure 2 shows the response frequency for each time bin as a function of context and segmentation task. To temporally align events, the order of situations in the noncontextual conditions was realigned with the order of situations in the contextual conditions. This allowed us to broadly compare the break points in the contextual and noncontextual conditions even though the length of the same situations across the two clips differed slightly, as they were filmed independently to avoid introducing film cuts. Figure 3 depicts the alignment of the two conditions.

Like Boggia and Ristic (2015), the contextual condition yielded time bins with unique social and nonsocial break points as well as time bins with overlapping social and nonsocial break points. In the contextual condition (Fig. 2, solid

\footnotetext{
${ }^{1} 15$-s bins were used for consistency with Boggia and Ristic (2015). However, a different study analyzing a subsection of the stimulus using 2-s bins showed similar results to the ones presented here (Capozzi, Latif, \& Ristic, 2019).
} 


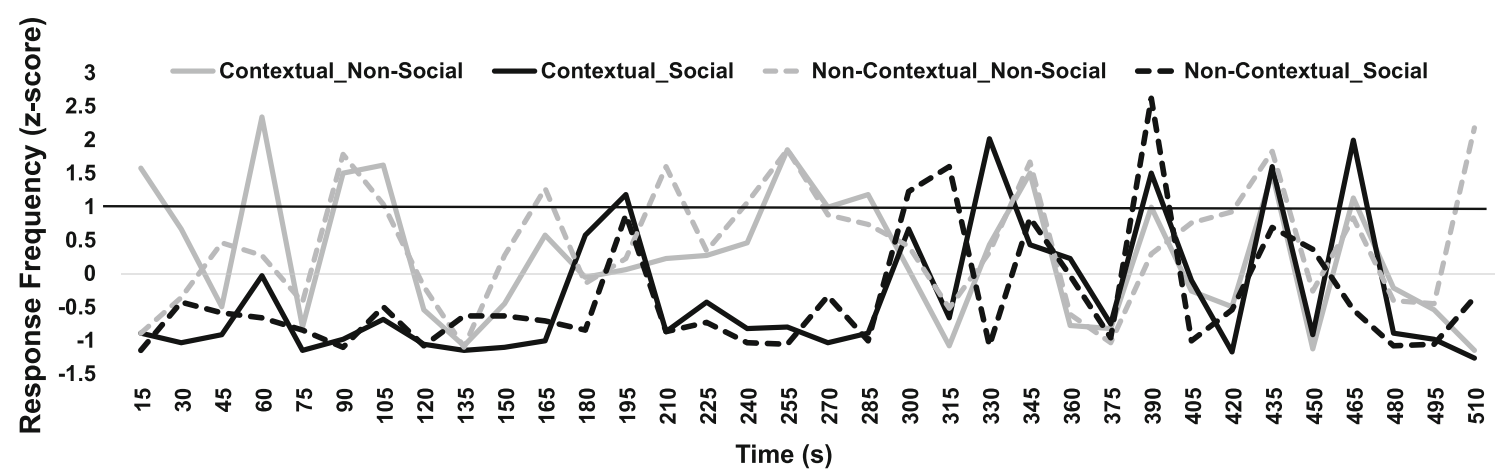

Fig. 2 Response frequency ( $z$ scores) as a function of context and task. The order of situations in the noncontextual condition has been temporally realigned with the order of situations in the contextual condition such that the start of each situation in the noncontextual condition matched the start of the same situation in the contextual condition. One (1) standard deviation is indicated by the solid dark line lines), nonsocial event break points were identified at $45 \mathrm{~s}$ to60 $\mathrm{s}^{2}{ }^{2} 75 \mathrm{~s}$ to $105 \mathrm{~s}$ (printer printing), $250 \mathrm{~s}$ to $285 \mathrm{~s}$ (door opening/actor entering), $330 \mathrm{~s}$ to $345 \mathrm{~s}$ (fax machine turning on/printing), $375 \mathrm{~s}$ to $390 \mathrm{~s}$ (door opening/actor entering), $420 \mathrm{~s}$ to $435 \mathrm{~s}$ (actor starting to play a game on his phone individually), and $450 \mathrm{~s}$ to $465 \mathrm{~s}$ (actor resuming to play on his phone individually after playing with the other actor). Social break points were identified at 180 s to $195 \mathrm{~s}$ (phone call to mom with message), $315 \mathrm{~s}$ to $330 \mathrm{~s}$ (phone call to mom), $375 \mathrm{~s}$ to $390 \mathrm{~s}$ (two actors playing on their phones together), $420 \mathrm{~s}$ to $435 \mathrm{~s}$ (one actor asking other actor to play on their phones together), and $450 \mathrm{~s}$ to $465 \mathrm{~s}$ (actors agreeing to cease playing on the phones together). The windows from $420 \mathrm{~s}$ to $435 \mathrm{~s}$ and $450 \mathrm{~s}$ to $465 \mathrm{~s}$ were identified as containing both social and nonsocial events. As such, these data, overall, are similar to those reported by Boggia and Ristic (2015).

In the noncontextual condition (Fig. 2, dashed lines), participants identified nonsocial break points at $75 \mathrm{~s}$ to $105 \mathrm{~s}$ (actor starting to play a game on his phone individually), $150 \mathrm{~s}$ to $165 \mathrm{~s}$ (printer printing), $195 \mathrm{~s}$ to $210 \mathrm{~s}$ (actor entering), $225 \mathrm{~s}$ to $255 \mathrm{~s}$ (actor exiting), $330 \mathrm{~s}$ to $345 \mathrm{~s}$ (fax machine turning on/ printing), $420 \mathrm{~s}$ to $435 \mathrm{~s}$ (one actor asking other actor to play on their phones together), and $495 \mathrm{~s}$ to $510 \mathrm{~s}$ (actor exits) and social break points at $285 \mathrm{~s}$ to $315 \mathrm{~s}$ (phone call to mom) and $375 \mathrm{~s}$ to $390 \mathrm{~s}$ (two actors playing on their phones together). It is important to note that despite some differences in the length between the contextual and noncontextual clips (i.e., 8:17 s [contextual] vs. 10:52 $\mathrm{s}$ [noncontextual]), the identified break points reflected many of the same situations within the clips as depicted in Fig. 3. That is, the content of 6 out of 8 nonsocial (i.e., $75 \%$ ) and 3 out of 5 (i.e., $60 \%$ ) social break points corresponded between contextual and noncontextual conditions. In other words, although the differences in clip lengths affected the start of some situations across the two contextual conditions, they did not affect the

\footnotetext{
${ }^{2}$ The event at this time bin was kettle boiling, which was removed from all subsequent analysis as it was not included in the noncontextual version of the video.
}

conceptual events that participants marked as containing event break points. This result provides additional support for the strength of the event segmentation process and its resilience to external factors such as temporal context. To quantify the strength of this behavior, we next evaluated the amount of response agreement within participants, or 'group agreement' in judging event boundaries (Zack \& Swallow, 2007).

\section{Group agreement}

We examined group agreement at all time bins in which the participants indicated an event boundary (i.e., when the response frequency for the bin exceeded one standard deviation from the group mean) relative to the total number of participants. Group agreement at breakpoints was entered in a $2 \times 2 \times 2$ mixed analysis of variance (ANOVA), with event type (social vs. nonsocial) and condition (contextual vs. noncontextual) included as within-subjects factors, and the order of contextual condition presentation (contextual first vs. noncontextual first) included as the between-subjects factor.

As illustrated in Fig. 4a, this analysis yielded a significant main effect of event type, $F(1,6)=23.71, p=.001, \eta_{\mathrm{p}}{ }^{2}=.80$, with greater group agreement for social $(M=.60, S E=.01)$ compared with nonsocial events $(M=.48, S E=.02)$. No significant effects of context, $F(1,6)=1.98, p=.21$, or any significant interactions were found (all $F \mathrm{~s}<1$ ), indicating that the availability of contextual information did not significantly impact group agreement in identifying either social or nonsocial events.

However, as plotted in Fig. 4b, the order of presentation influenced the results, such that response agreement was higher in the group of participants who viewed contextual clip first $(N=100 ; M=.57, S E=.007)$ compared with the response agreement of the group of participants who viewed the noncontextual clip first $(N=95 ; M=.52, S E=.007)$, main effect $F(1,6)=25.29, p=.002, \eta_{\mathrm{p}}^{2}=.80$. Thus, viewing the clip within the appropriate context first facilitated response 
Non-Contextual

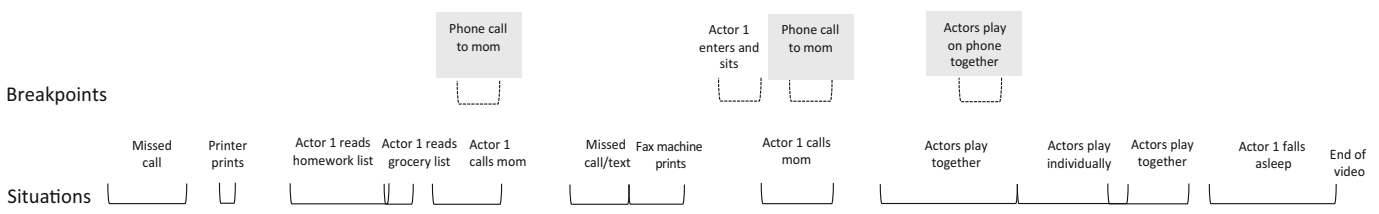

Contextual
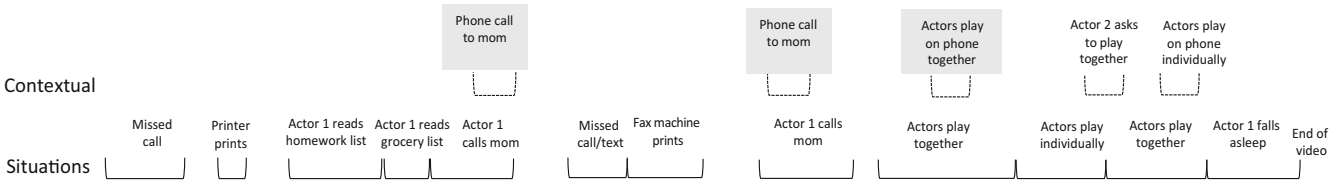

Non-Social Event Breakpoints

\section{Non-Contextual}
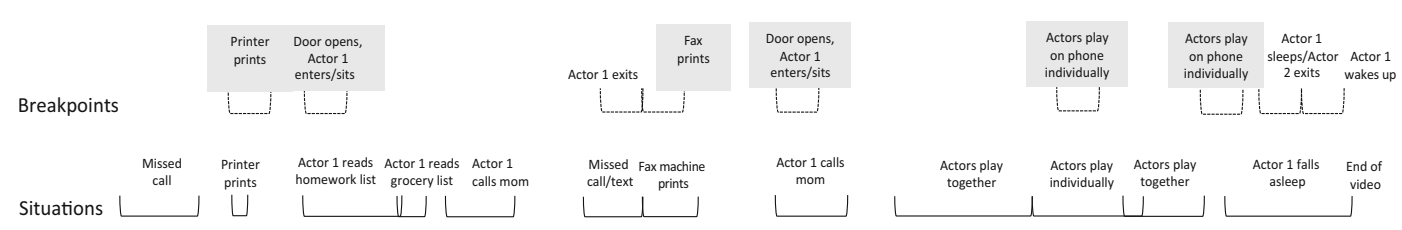

Contextual
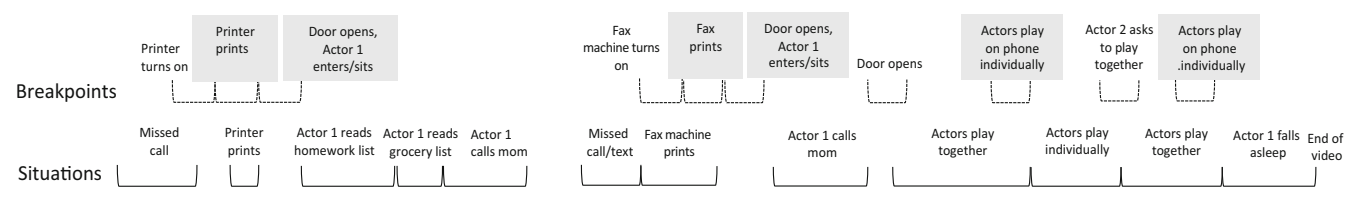

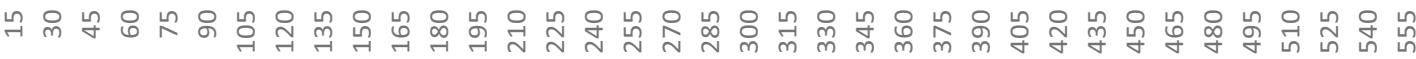 \\ Time (s)}

Fig. 3 Clip timing, content, and break points for contextual and noncontextual conditions. The order of situations in the noncontextual condition has been temporally realigned with the order of situations in the contextual condition such that the start of each situation in the

agreement at breakpoints later. No other effects or interactions were reliable (all $F \mathrm{~s}<1$ ).

To sum, our first question asked whether social segmentation was affected by the removal of the clip content's appropriate temporal sequence. We found that participants identified break points consistently in both appropriate and scrambled contexts with the availability of coherent context facilitating increased response agreement at break points in both social and nonsocial segmentation tasks when the contextual version of the video was presented first. That is, more participants agreed on event boundaries when they viewed the clip within the coherent context first.

Next, we addressed our second question in which we examined whether the lack of context modulated perceivers' reliance on available perceptual visual and auditory cues during segmentation behaviour. To do so, we conducted motion and auditory signal analyses on the clips and related their noncontextual condition matched the start of the same situation in the contextual condition. The grey squares denote break points that matched in content across the two conditions

indices to segmentation behaviour. If visual and/or auditory content influenced participants' perception of event boundaries, we expected to find a significant difference in the auditory and visual content in the time bins corresponding to event break points relative to the time bins that did not contain event break points. Further, we also expected to find a higher correlation between the strength of visual and auditory content and the frequency of participant key presses ( $z$ scores).

\section{Visual and auditory signal analysis}

Motion Motion was analyzed as an instance of visual information. Variation in motion information was quantified using optical flow analysis (OFA), a standardized computer-vision technique (Horn $\&$ Schunck, 1981). Optical flow refers to the pattern of movement of low-level features such as edges, textures, and surfaces relative to 
an observer. In optical flow analysis, the video camera is the theoretical 'observer' and the patterns of motion are inferred by comparing the patterns of pixel intensity between consecutive frames. The differences in pixel intensities between frames results in a series of pixel velocities that can be summed and correspond to the magnitude of motion change. Measures of motion magnitude across all frames provides a time series of total motion as it changes over the course of the video. Here, we performed OFA using FlowAnalyzer (Barbosa, 2017; https://www.cefala.org/FlowAnalyzer/). FlowAnalzyer uses the Horn and Schunck (1981) method to estimate motion by detecting the boundaries of a moving object and assuming a smooth motion trajectory from one video frame to the next. FlowAnalyzer is a validated algorithm for the calculation of the Horn and Schunck (1981) estimation of optical flow (Danner, Barbosa, \& Goldstein, 2018; K. C. Hall, Allen, McMullin, Letawsky, \& Turner, 2015). We computed the average motion within the same 15-s bins that were used to analyze response $z$ scores above, and $p$ values were corrected formultiple comparisons using Bonferroni where appropriate.

To examine whether overall motion influenced participants' key presses, we computed Pearson correlations between the frequency of key presses ( $z$ scores) and the average motion signal calculated at each of the same 15-s time bins used for the event segmentation task. This analysis was carried out separately for social and nonsocial event segmentation tasks and for each contextual and noncontextual condition. ${ }^{3}$ If signal motion differences at event break point bins influenced segmentation behaviour, we expected to find a stronger correlation between fluctuations in motion and the $z$ scores. Our results supported this hypothesis for social event segmentation only. That is, as plotted in Fig. 5a-b, we found a significant correlation between overall motion and $z$ scores for social segmentation in both contextual, $r(33)=.52, p=.001$, and noncontextual conditions, $r(33)=.43, p=.01$. The correlation between motion and $z$ scores was not significant for nonsocial segmentation, $r(33)=.26, p=.07$, contextual; $r(33)=.06, p=$ .74 , noncontextual. It is important to note that this effect reflects the influence of overall motion rather than variability in motion within the bins, as confirmed by correlations between the standard deviation of motion

\footnotetext{
${ }^{3}$ We examined if average motion in the bins containing break points was different than average motion in bins containing no break points. For the contextual condition, the average motion in bins containing event break points was numerically greater $(M=15.07, S E=.65)$ than average motion in the bins not containing event break points $(M=12.43, S E=1.02), t(31)=2.28, p=.06$, $\eta^{2}=.15$, for the social events but not the nonsocial events, $t(31)=.109, p=$ .56 , although this difference did not reach significance. The same pattern of results was found for the noncontextual condition where there was a trending difference in the average motion for social events $(M=16.33, S E=1.03)$ compared with bins not containing break points $(M=12.95, S E=.62), t(31)$ $=2.20, p=.07, \eta^{2}=.14$. No effects emerged for the non-social events $(t(31)=.12, p=1.0)$.
}
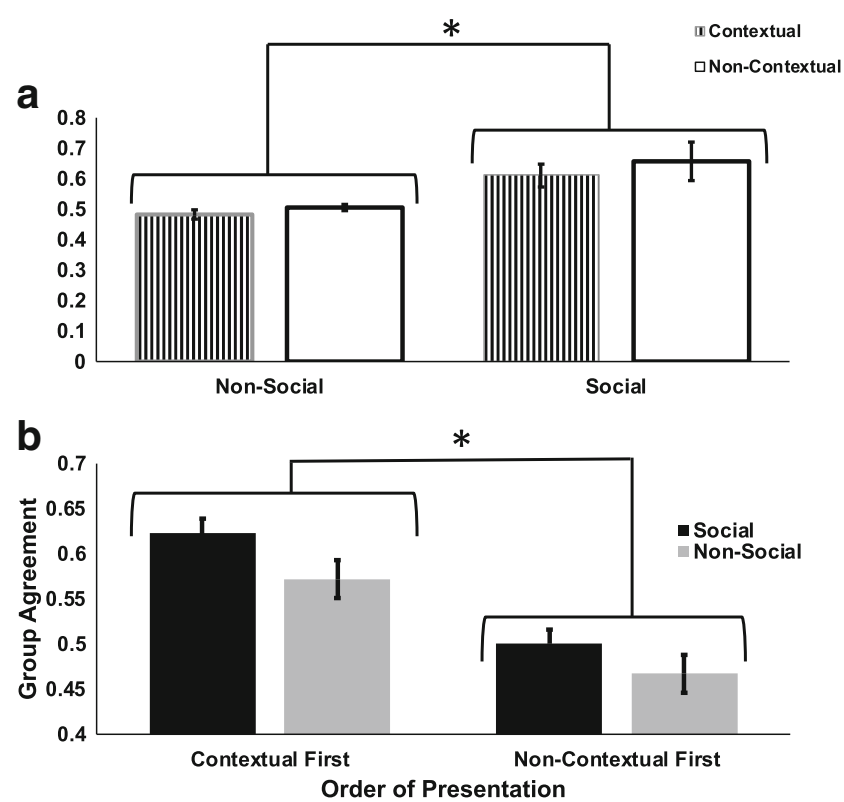

Fig. 4 Average group agreement (in proportions) for social and nonsocial events as a function of context (a) and order of condition presentation (b). Errors Bars denote standard error.

signal within each bin and the frequency of responses ( $z$ scores). After corrections for multiple comparisons, there were no significant relationships for nonsocial, $r(33)=.36$, contextual; $r(33)=.32$, noncontextual, or social segmentation, $r(33)=.32$, contextual; $r(33)=$ .26 , noncontextual.

Thus, the analyses of optical flow within the clip found that segmenting social, but not nonsocial events, was related to the amount of motion signal in the environment. However, dovetailing with our behavioral group agreement data, this result was not specific to contextual condition, which also showed greater agreement for social events with little effect of context.

Auditory signal Sound amplitude (i.e., energy/intensity in an auditory signal) was examined as a general index of auditory information. Amplitude refers to the height or the energy of a sound wave and typically corresponds to the perceptual experiences of loudness of an auditory signal as measured in decibels (dB). It should be noted that other factors (e.g., frequency of the sound) also correspond to the experience of loudness; however, amplitude was selected, as it has been previously used as a valid index of social auditory information (e.g., Foulkes, Docherty, Khattab, \& Yaeger-Dror, 2010; Tusing \& Dillard, 2000). To extract sound amplitude, we used Praat, a software designed for acoustic analysis (Boersma, 2001). We set the sampling at $0.01 \mathrm{~s}$ and used the built-in 'get Intensity' command. To directly relate variation in sound availability to event segmentation, we averaged the sound amplitude within the same 15-s bins as the event segmentation task. 

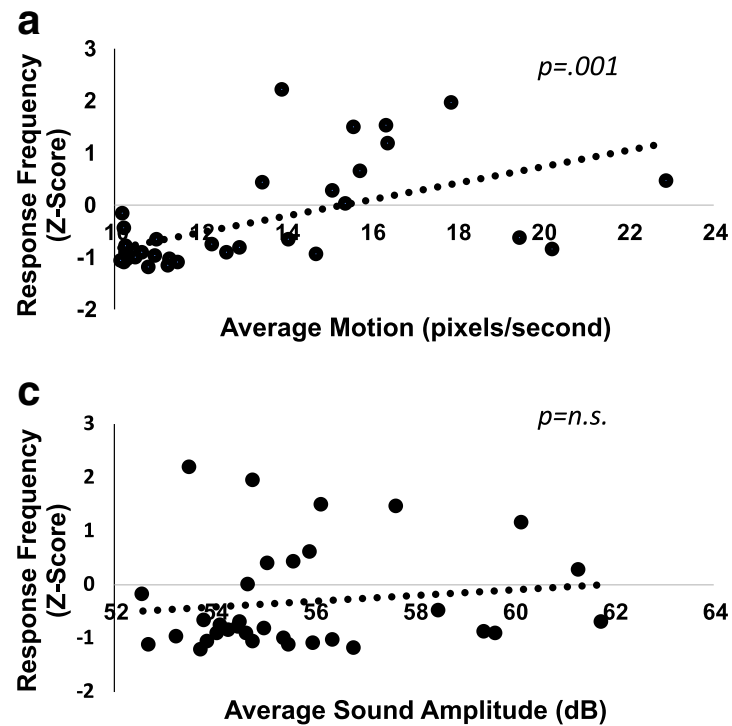

Fig. 5 Correlation between average motion and frequency of responses for the contextual (a) and noncontextual (b) conditions for social event segmentation. Correlation between average sound amplitude and

To determine whether the sound amplitude differences in event break point bins influenced participants' key presses, we computed Pearson correlations between the auditory signal and $z$ scores for all bins separately for social and nonsocial event segmentation in both contextual conditions. ${ }^{4}$ If sound information contributed to event segmentation, we expected to find a significant correlation between sound fluctuations and response frequency ( $z$ scores). As shown in Fig. $5 \mathrm{c}$, there was a significant correlation between the auditory signal and $z$ scores, $r(33)=.44, p=.009$, for social events in the noncontextual condition. No reliable relationship was found in the other conditions (see Fig. 5d). Thus, when context was not available, response frequency was significantly related to the intensity of the auditory signal, but only for nonsocial segmentation. As with the motion signal, it was the overall sound intensity that influenced segmentation behaviour rather than the variability in sound amplitude, as once again no significant correlations between the standard deviation of responses and average amplitude emerged for both nonsocial, $r(33)=-.05$, contextual; $r(33)=-.06$, noncontextual, and social segmentation, $r(33)=.17$, contextual; $r(33)=-.12)$, noncontextual.

\footnotetext{
${ }^{4}$ Like visual signal, here we examined if sound cues in the bins containing break points were different than sound cues in the bins without break points. For the contextual condition, we did not find any significant difference in sound amplitude in event break-point bins compared with non-break-point bins for either the social, $t(31)=1.011, p=.64$, or the nonsocial events, $t(31)=.36, p=1.0$. In the noncontextual condition, however, we found that the average sound amplitude in bins containing social event break points was numerically greater $(M=57.55, S E=.89)$ than sound amplitude in the bins not containing social event break points $(M=55.41, S E=.49), t(31)=2.26, p=$ $.06, \eta^{2}=.14$, although this difference did not reach significance. This pattern was not observed for the nonsocial events, $t(31)=.57, p=1.0$.
}
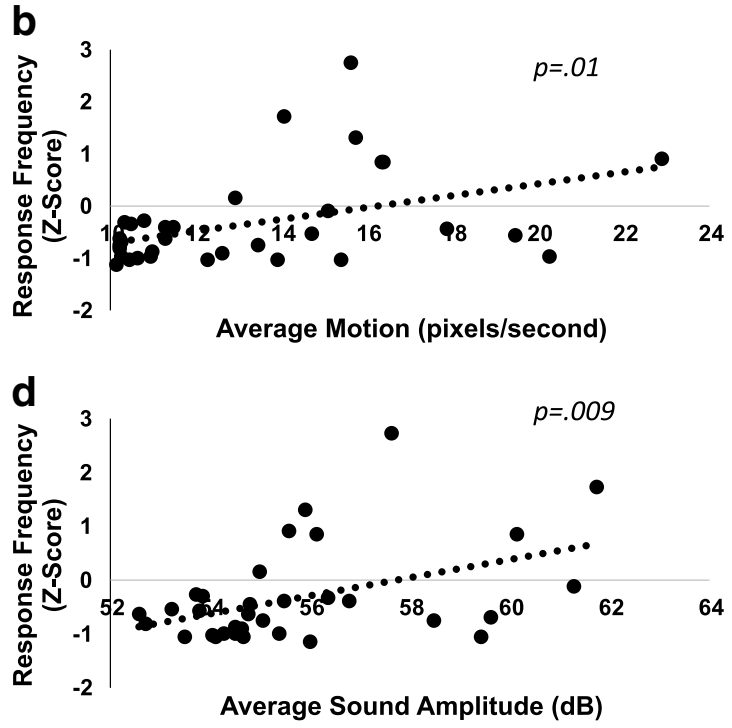

frequency responses for the contextual (c) and noncontextual (d) conditions for social event segmentation

Overall then, the analyses of motion and auditory perceptual information showed that, for social segmentation specifically, the motion signal was reliably correlated with group response agreement in both contextual and noncontextual conditions while the intensity of sound was related to group response agreement in the noncontextual case only. This suggests that, although context did not determine social segmentation behaviour, it differentially modulated participants' reliance on available auditory and visual perceptual cues.

\section{Discussion}

In this study we examined how the availability of temporal contextual information within the environment influenced social event segmentation. The role of context has generally been shown to be important for accurate interpretation of social information (Barrett, Mesquita, \& Gendron, 2011; Trope, 1986), however, so far, its specific role in social event segmentation was not investigated. Here, we demonstrated that the broad context within which social events occur did not determine but rather modulated observer response agreement and reliance on sensory information when segmenting social events.

Overall, our results replicated previous findings showing greater agreement for social, compared with nonsocial events (Boggia \& Ristic, 2015). Temporal context, however, did not appear to determine either social or nonsocial events. Although participants indicated some break points within different time bins in contextual and noncontextual conditions due to slightly different clip timing, they nevertheless identified break points in many of the same situations within the two 
clips. Identifying similar situations within the clips independently of the clip's temporal context lends further support for the notion that a coherent story line does not determine segmentation or group agreement, as participants indicated breakpoints with similar amounts of agreement regardless of whether the situations were presented within a coherent story line or not. This finding is consistent with previous studies that indicated that participants identified similar nonsocial events when they viewed them in the correct order compared with when viewing the same scene in reverse order (Hard et al., 2008).

Our finding that the clip's contextual information did not drive social event segmentation is intriguing considering the importance of context in the interpretation of social behaviour (Barrett et al., 2011; Trope, 1986). This result is broadly consistent with previous work that has demonstrated the hierarchical nature of event perception. In particular, when understanding complex and unpredictable situations that contain both social and nonsocial events, humans rely more on the specific events within a broader context than the overall context (Zacks, 2004). Such a distinction in the specific versus broad use of information when understanding complex situations has also been found at a neural level, suggesting distinct activity related to each kind of grouping of dynamic information (Zacks et al., 2001). Interestingly, a similar hierarchical organization has also been implicated in research on narrative comprehension where specific brain activity is associated with different time scales of understanding of a story line, with, for example, differential activity found when understanding sounds versus words versus sentences versus overall narrative (Lerner, Honey, Silbert, \& Hasson, 2011). Our findings show that not only do observers rely on smaller perceptual information more than larger context, they do not appear to require the overall context in order to segment ongoing dynamic social and nonsocial activity.

Although context generally did not determine identification of social and nonsocial events, contextual information influenced event segmentation behaviour to some extent. In particular, when participants viewed the contextual version of the video first, they exhibited increased response agreement in identifying both social and nonsocial break points. The importance of the event order in the understanding of social situations has been well established. A coherent ordering of events allows for a more straightforward evaluation of the importance and value of social behaviour while determining causal relationships between events (Gergen \& Gergen, 1988; Münte, Schiltz, \& Kutas, 1998). Thus, having access to coherent ordering of events influences later interpretation of those events. For example, when participants are presented events in an atypical order, their subsequent memory (Bower, Black, \& Turner, 1979) and their comprehension (Ohtsuka \& Brewer, 1992) for those events is less accurate than when presented in a coherent ordering. This dovetails with the present results, whereby viewing the events within a coherent storyline first facilitated subsequent event segmentation.

Our results further revealed that segmentation behaviour was also related to the dynamics of perceptual information within the videos. This is consistent with studies which have demonstrated an important role of perceptual information in event segmentation even in the presence of strong top-down effects such as observer preference bias (such as segmenting videos of one's own favourite sports team; Huff et al., 2017). Here, we found clear perceptual effects in that participants' responses to social, but not nonsocial, events were reliably related to the dynamics of motion within the videos. This is consistent with previous work that has highlighted the importance of movement in the understanding of social cues (J. A. Hall, Hogan, \& Murphy, 2018). Here, the reliance on motion information occurred regardless of the availability of context. However, when context was not available, participants pattern of responses to the social events was also reliably related to the dynamics of auditory information. This might suggest that when there is uncertainty in interpretation of social information, participants reweigh their reliance on specific perceptual cues needed to perform social segmentation. This notion is consistent with work in social perception whereby when the reliability of information is questionable or if the information is noisy, in order to compensate, participants actively start modifying their use of available information (Boyle, Kayser, \& Kayser, 2017). As such, our findings also highlight the importance of context as a modulator of perceptual information usage.

Further, since motion and auditory analysis revealed no relationships with nonsocial segmentation, it is important to consider what other sources of information might be driving this process. First, it is possible that social events, overall, contain more perceptual information relative to nonsocial events. Although this may suggest that humans use available perceptual information differently to identify social events, it may also suggest that segmentation of nonsocial events may be related to the amount of perceptual information available. This question remains to be addressed in future research. Another possibility is that our measures of motion and sound were not conducive to capturing the variations in nonsocial content. Both motion and auditory variations play a salient and complex role within the social domain (Robins, Hunyadi, \& Schultz, 2009) such that humans are experts at perceiving and integrating this information to make important social inferences (Zaki, 2013). As such, this analysis may be more conducive for capturing variations in perceptual information that is most relevant for social, but not nonsocial segmentation, with the segmentation of nonsocial events relying on low-level changes in static perceptual features (e.g., colours, shapes, simple tones; Zacks, 2004; Zacks et al., 2006) or more specific and targeted bodily movements 
(Endres, Christensen, Omlor \& Giese, 2011) that was not assessed by the present analyses. Further work is needed to investigate whether there are differences the nature of perceptual cues that are used to perform social versus nonsocial segmentation.

The data presented here open several additional future research avenues. One, while we used a temporal context manipulation here, future investigations could explore whether other forms of contextual disruption such as incompatible semantic context or events that lack meaning, may affect social event segmentation (e.g., Galleguillos \& Belongie, 2010). Further, it is also possible that the temporal disruption influences social segmentation on a finer scale. Thus, one could investigate whether contextual information modulates social and nonsocial segmentation when context is manipulated at a finer time scale, such as by scrambling the order of more fine-grained situations. This question is pertinent because previous work in neuroimaging has identified that humans understand narratives on several timescales (Lerner et al., 2011), and as such it is possible that a similar hierarchical neural mechanism may also be in place during event segmentation. Relatedly, further investigation of how observers may categorize events into 'fine' versus 'coarse' events (e.g., Zacks \& Swallow, 2016) may contribute to our understanding of event segmentation, given observers may be sensitive to both a temporal and grouping hierarchy. Two, an additional line of inquiry could examine if context matters differently when participants observe naturalistic behaviour versus when they experience it in real world or directly act within an environment. This is because many recent studies point to large differences between behaviour measured in the laboratory versus the real world (Foulsham, Walker, \& Kingstone, 2011), and consequently the mechanisms underlying event segmentation may be different when one observes versus directly acts on the environment. Further, events in different contexts may derive different goals. Given that generally actors' goals contribute to event segmentation (Magliano, Miller, \& Zwaan, 2001), it would be important to investigate how context and observed behaviour, as driven by actor goals, would contribute to the grouping of events. Finally, future work is needed to understand more precisely which aspects of visual and auditory information observers rely on during event segmentation. Specifically, while the present analyses indicate differential roles of perceptual information with reduced contextual information, it remains to be determined which aspects of motion (e.g., a more fine-grained motion analysis quantifying specific gestural and head motion) and which aspects of the auditory signal (e.g., other social vocal cues such as prosody) may be key in this process.

In sum, the results of this work show that like nonsocial event segmentation, social event segmentation also does not depend on, but may be modulated by, the larger temporal context. They also show that once temporal context is removed, participants begin to use visual and auditory perceptual cues within the clip differently during social, relative to nonsocial, segmentation. This shows that social segmentation is a flexible process that benefits both from the available context and multiple perceptual signals within the environment.

Acknowledgments This research was supported by the Social Sciences and Humanities Research Council of Canada (SSHRC), the Natural Sciences and Engineering Council of Canada (NSERC), the William Dawson Chairs Fund, and the Fonds du Recherche du Québec-Société et Culture.

Open practices statement Experimental code used to run this study is available through the open software link in the manuscript. Data for this experiment can be made available upon request.

\section{References}

Anderson, B. L., \& Winawer, J. (2005). Image segmentation and lightness perception. Nature, 434, 79-83.

Barbosa, A. V. (2017). FlowAnalyzer [Computer software]. Retrieved from https://www.cefala.org/FlowAnalyzer/

Barrett, L. F,, Mesquita, B., \& Gendron, M. (2011). Context in emotion perception. Current Directions in Psychological Science, 20(5), 286-290.

Boersma P (2001). Praat, a system for doing phonetics by computer. Glot International 5:9/10, 341-345.

Boggia, J., \& Ristic, J. (2015). Social event segmentation. The Quarterly Journal of Experimental Psychology, 68(4), 731-744.

Bower, G. H., Black, J. B., \& Turner, T. J. (1979). Scripts in memory for text. Cognitive Psychology, 11(2), 177-220.

Boyle, S. C., Kayser, S. J. \& Kayser, C. (2017). Neural correlates of multisensory reliability and perceptual weights emerge at early latencies during audio-visual integration. The European Journal of Neuroscience, 46, 2565-2577.

Capozzi, F., Latif, N., \& Ristic, J. (2019). It's not all in the face: Reduced face visibility does not modulate social segmentation. Visual Cognition https://doi.org/10.1080/13506285.2019.1579774

Christopher A. Kurby, Jeffrey M. Zacks, (2008) Segmentation in the perception and memory of events. Trends in Cognitive Sciences $12(2): 72-79$

Danner, S. G., Barbosa, A. V., \& Goldstein, L. (2018). Quantitative analysis of multimodal speech data. Journal of Phonetics, 71, 268-283.

de Gelder, B., \& Vroomen, J. (2000). The perception of emotions by ear and by eye. Journal of Cognition and Emotion, 14(3), 289-311.

de Leeuw, J. R. (2015). jsPsych: A JavaScript library for creating behavioural experiments in a Web Browser. Behavior Research Methods, $47,1-12$.

Dominik Endres, Andrea Christensen, Lars Omlor, Martin A. Giese, (2011) Emulating human observers with bayesian binning. ACM Transactions on Applied Perception 8 (3):1-12

Foulkes, P., Docherty, G. J., Khattab, G., \& Yaeger-Dror, M. (2010). Sound judgements: Perception of indexical features in children's speech. In D. Preston \& N. Niedzielski (Eds.), A reader in sociophonetics (pp. 327-356). Berlin, Germany: Mouton de Gruyter. 
Foulsham, T., Walker, E., \& Kingstone, A. (2011). The where, what and when of gaze allocation in the lab and the natural environment. Vision Research, 51(17), 1920-1931.

Galleguillos, C., \& Belongie, S. (2010). Context based object categorization: A critical survey. Computer Vision and Image Understanding, 114(6), 712-722.

Gergen, K. J., \& Gergen, M. M. (1988). Narrative and the self as relationship. Advances in Experimental Social Psychology, 21, 17-56.

Hall, J. A., Hogan, T. G., \& Murphy, N. A. (2018). Nonverbal communication. Annual Review of Psychology, 70(24). https://doi.org/10. 1146/annurev-psych-010418-103145

Hall, K. C., Allen, C., McMullin, K., Letawsky, V., \& Turner, A. (2015). Measuring magnitude of tongue movement for vowel height and backness. Paper presented at the Proceedings of the International Congress of Phonetic Sciences, Glasgow, UK.

Hard, B. M., Tversky, B., \& Lang, D. (2008). Making sense of abstract events: Building event schemas. Memory \& Cognition, 34(6), 12211235.

Horn, B. K. P., \& Schunck, B. G. (1981). Determining optical flow. Artificial Intelligence, 17(1/3), 185-203.

Huff, M., Papenmeier, F., Maurer, A. E., Meitz, T. G. K., Garsoffsky, B., \& Schwan, S. (2017). Fandom biases retrospective judgments not perception. Scientific Reports, 7, 43083. https://doi.org/10.1038/ srep43083

Jeffrey M. Zacks, Khena M. Swallow, (2016) Event Segmentation. Current Directions in Psychological Science 16 (2):80-84

Lerner, Y., Honey, C. J., Silbert, L. J., \& Hasson, U. (2011). Topographical mapping of a hierarchy of temporal receptive windows using a narrated story. The Journal of Neuroscience, 31(8), 2906-2915.

Magliano, J. P., Miller, J., \& Zwaan, R. A. (2001). Indexing space and time in film understanding. Applied Cognitive Psychology, 15(5), $533-545$.

Magliano, J. P., \& Zacks, J. M. (2011). The impact of continuity editing in narrative film on event segmentation. Cognitive Science, 35, 1489 1517.

Munhall, K. G., Jones, J. A., Callan, D. E., Kuratate, T., \& VatikiotisBateson, E. (2004). Visual prosody and speech intelligibility: Head movement improves auditory speech perception. Psychological Science, 15(2), 133-137.

Münte, T. F., Schiltz, F., \& Kutas, M. (1998). When temporal terms belie conceptual order. Nature, 395, 71-73.
Newtson, D., \& Engquist, G. (1976). The perceptual organization of ongoing behavior. Journal of Experimental Social Psychology, 12(5), 436-450.

Ohtsuka, K., \& Brewer, W. F. (1992). Discourse organization in the comprehension of temporal order in narrative texts. Discourse Processes, 15(3), 317-336.

Pelphrey, K. A., Morris, J. P., \& McCarthy, J. (2004). Grasping the intentions of others: The perceived intentionality of an action influences activity in the superior temporal sulcus during social perception. Journal of Cognitive Neuroscience, 16(10), 1706-1716.

Robins, D. L., Hunyadi, T., \& Schultz, R. T. (2009). Superior temporal activation in response to dynamic audio-visual cues. Brain and Cognition, 69(2), 269-278.

Speer, N. K., Reynolds, J.R., \& Zacks, J. M. (2007). Human brain activity time-locked to narrative event boundaries. Psychological Science, 18(5), 449-455.

Trope, Y. (1986). Identification and inference processes in dispositional attribution. Psychological Review, 93, 239-257.

Tusing, K. J., \& Dillard, J. P. (2000). The sounds of dominance: Vocal precursors of perceived dominance during interpersonal influence. Human Communication Research, 26(1), 148-171.

Wurm, M. F., \& Schubotz, R. I. (2012). Squeezing lemons in the bathroom: Contextual information modulates action recognition. NeuroImage, 59(2), 1551-1559.

Zacks, J. M. (2004). Using movement and intentions to understand simple events. Cognitive Science, 28(6), 979-1008.

Zacks, J. M., Braver, T. S., Sheridan, M. A., Donaldson, D. I., Snyder, A. Z., Ollinger, J. M., . . . Raichle, M. E. (2001). Human brain activity time-locked to perceptual event boundaries. Nature Neuroscience, 4(6), 651-655.

Zacks, J. M., Swallow, K. M., Vettel, J. M., \& McAvoy, M. P. (2006). Visual motion and the neural correlates of event perception. Brain Research, 1076, 150-162

Zaki, J. (2013). Cue integration: A common framework for social cognition and physical perception. Perspectives on Psychological Science, 8(3), 296-312.

Publisher's note Springer Nature remains neutral with regard to jurisdictional claims in published maps and institutional affiliations. 